\title{
Four-jet double parton scattering production in proton-nucleus collisions within the PYTHIAs framework
}

\author{
Oleh Fedkevych $\oplus^{*}$ \\ Dipartimento di Fisica, Università di Genova, \\ Via Dodecaneso 33, 16146 Genoa, Italy \\ Leif Lönnblad ${ }^{\dagger}$ \\ Department of Astronomy and Theoretical Physics, \\ Sölvegatan 14A, S-223 62 Lund, Sweden
}

(Received 14 February 2020; accepted 29 June 2020; published 17 July 2020)

\begin{abstract}
We present our studies of four-jet double parton scattering production in proton-nucleus collisions within the framework of the PYTHIA8 event generator. We demonstrate that double absorptive processes in $\mathrm{p} A$ generated by the Angantyr model in PYTHIA8 give an enhancement of the total double parton scattering cross section similar to the predictions by Strikman and Treleani in 2001. Additionally, we discuss how the growth of activity in the direction of a nucleus affects an $A$ scaling of a total double parton scattering cross section in proton-nucleus collisions.
\end{abstract}

DOI: 10.1103/PhysRevD.102.014029

\section{INTRODUCTION}

Despite a significant progress in both theoretical and experimental studies of QCD, many of its aspects still require further detailed investigation. One of the possible keys to a deeper understanding of QCD and a structure of hadrons is the study of so-called double parton scattering (DPS), a process when two hard interactions occur in a single hadron-hadron collision. Various studies of DPS performed at proton-proton ( $\mathrm{pp}$ ) and proton-antiproton $(\mathrm{p} \overline{\mathrm{p}})$ colliders [1-21] suggest the presence of partonic correlations which leads to small values of the effective DPS interaction area, $\sigma_{\text {eff }}$. The nature of these correlations is still under debate and is obscured by the difficulties involved with disentangling different sources of parton correlations. As a tool for gaining further insights, Strikman and Treleani proposed to study DPS processes in proton-nucleus ( $\mathrm{pA}$ ) collisions [22] which would allow the separation of transverse from longitudinal parton correlations according to the different $A$ dependence of the corresponding contributions to a total DPS cross section. This idea got a further development in [23-27] and found some phenomenological applications in a series of works [23-25,28-35].

\footnotetext{
*oleh.fedkevych@ge.infn.it

${ }^{\dagger}$ leif.lonnblad@thep.lu.se
}

Published by the American Physical Society under the terms of the Creative Commons Attribution 4.0 International license. Further distribution of this work must maintain attribution to the author(s) and the published article's title, journal citation, and DOI. Funded by SCOAP ${ }^{3}$.
While a significant progress in a theoretical description of DPS in $\mathrm{p} A$ collisions has been achieved, it is important to also have a framework for realistic simulations of DPS in $\mathrm{p} A$ collisions to be able to verify it experimentally. In this paper, we compare predictions of the Strikman and Treleani model against predictions of the Angantyr model of $\mathrm{p} A$ collisions [36] recently implemented in the PYTHIA8 event generator [37,38]. Since dependence of a DPS cross section on the total number of nucleons predicted by Strikman and Treleani is based upon general geometrical properties of a nuclear target we argue that every model of DPS in $\mathrm{p} A$ collisions should exhibit a similar $A$ dependence.

We discuss in detail the differences and similarities between the models and demonstrate that the Angantyr model gives predictions similar to those given by Strikman and Treleani, and can therefore be used to simulate the predictions in an experimental setting.

Since Angantyr takes advantage of the entire PYTHIA machinery, including multiple parton interaction (MPI), initial and final state radiation, and many other effects, we therefore conclude that it can be used to give standalone Monte Carlo simulations of complete four-jet DPS production events in $\mathrm{p} A$ collisions.

This paper is organized as follows: in Secs. II and III, we briefly sketch out the Strikman and Treleani and Angantyr models, respectively, in Sec. IV, we provide our simulations for the four-jet DPS production in $\mathrm{p} A$ collisions and compare them against predictions made within the framework of Strikman and Treleani, and in Sec. V, we summarize our results and discuss some further perspectives of DPS modeling in $\mathrm{p} A$ collisions. 


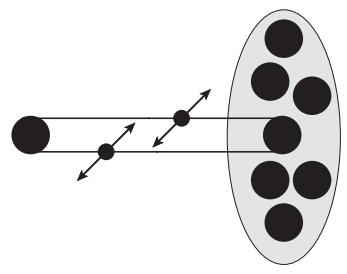

(a)

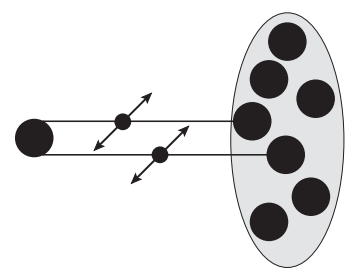

(b)
FIG. 1. A schematic representation of some possible DPS processes in $\mathrm{p} A$ collision. (a) DPS occur between one incident proton and one nucleon. (b) DPS occur between one incident proton and two different nucleons.

\section{STRIKMAN AND TRELEANI MODEL}

The composite nature of a nuclear target leads to various DPS contributions which are absent in pp $(\mathrm{p} \overline{\mathrm{p}})$ collisions. Apart from the "standard" DPS process shown in Fig. 1(a), one can have a DPS process involving one incident proton and two different nucleons, as schematically shown in Fig. 1(b). In the following, we will refer to processes in Figs. 1(a) and 1(b) as to DPS I and DPS II contributions, respectively.
Since DPS I and DPS II contributions involve different number of nucleons, it is quite natural to expect a different dependence of the corresponding total cross sections on an atomic mass number [39] $A$. In 2001, Strikman and Treleani published a pioneering work [22] where the expression for the total cross sections for DPS I and DPS II processes was given for the first time. Within their model, a total DPS cross section for $\mathrm{p} A$ collisions, assuming no interference between both DPS processes (which allows to consider a nucleus as a superposition of independent nucleons), can be written as a sum of two terms

$$
\sigma_{\mathrm{p} A}^{\mathrm{DPS}}=\sigma_{\mathrm{I}}^{\mathrm{DPS}}+\sigma_{\mathrm{II}}^{\mathrm{DPS}},
$$

where $\sigma_{\mathrm{I}}^{\text {DPS }}$ can be expressed, neglecting a difference between proton and neutron, in terms of a total DPS cross section for pp collisions as

$$
\sigma_{\mathrm{I}}^{\mathrm{DPS}}=A \sigma_{\mathrm{pp}}^{\mathrm{DPS}},
$$

where $\sigma_{\mathrm{pp}}^{\mathrm{DPS}}$ is a corresponding DPS cross section in pp collisions [41]. Neglecting quantum correlations and interference between partons, one can express $\sigma_{\mathrm{pp}}^{\mathrm{DPS}}$ as

$$
\sigma_{\mathrm{pp}}^{\mathrm{DPS}}=\frac{1}{1+\delta_{a b}} \sum_{a_{1}, a_{2}, b_{1}, b_{2}} \int \prod_{i=1}^{4} d x_{i} d^{2} b \Gamma_{a_{1}, a_{2} / h_{a}}\left(x_{1}, x_{2}, \boldsymbol{b}, Q_{1}, Q_{2}\right) \Gamma_{b_{1}, b_{2} / h_{b}}\left(x_{3}, x_{4}, \boldsymbol{b}, Q_{1}, Q_{2}\right) \hat{\sigma}_{a_{1}, b_{1} \rightarrow a} \hat{\sigma}_{a_{2}, b_{2} \rightarrow b},
$$

where the sum runs over parton flavors contributing to production of final states $a$ and $b$. The objects $\Gamma_{i, j / h}\left(x_{i}, x_{j}, \boldsymbol{b}, Q_{i}, Q_{j}\right)$ are called generalized two parton distribution functions (gPDFs), which in the first approximation can be seen as a probability to find two partons $i, j$ with longitudinal momentum fractions $x_{i}, x_{j}$ separated by transverse distance $|\boldsymbol{b}|$ in a given hadron $h$. We use factor $1+\delta_{a b}$ in denominator in order to reflect the fact that one has to divide a total cross section by 2 for production of two indistinguishable final states $a$ and $b$. In the following, in order not to overload our notation, in Eq. (3), we skip dependence on factorization scales $Q_{1}, Q_{2}$ as well as hadron labels $h_{a}, h_{b}$.

Assuming that in $\mathrm{gPDFs}$ one can approximately factorize dependence on longitudinal and transverse degrees of freedom

$$
\Gamma_{i, j}\left(x_{i}, x_{j}, \boldsymbol{b}\right) \approx D_{i, j}\left(x_{i}, x_{j}\right) F(\boldsymbol{b}),
$$

we can integrate out the $\boldsymbol{b}$ dependence in Eq. (3), which allows us to write

$$
\begin{aligned}
\sigma_{\mathrm{pp}}^{\mathrm{DPS}}= & \frac{1}{\sigma_{\mathrm{eff}}} \frac{1}{1+\delta_{a b}} \sum_{a_{1}, a_{2}, b_{1}, b_{2}} \int \prod_{i=1}^{4} d x_{i} d^{2} b D_{a_{1}, a_{2}}\left(x_{1}, x_{2}, \boldsymbol{b}\right) \\
& \times D_{b_{1}, b_{2}}\left(x_{3}, x_{4}, \boldsymbol{b}\right) \hat{\sigma}_{a_{1}, b_{1}} \hat{\sigma}_{a_{2}, b_{2}}
\end{aligned}
$$

where we refer to the objects $D_{i, j}\left(x_{i}, x_{j}\right)$ as to the double parton distribution functions (dPDFs). The parameter $\sigma_{\text {eff }}$ in Eq. (5) is defined as

$$
\sigma_{\mathrm{eff}}=\left[\int d^{2} b F^{2}(\boldsymbol{b})\right]^{-1}
$$

and can be seen as an effective DPS interaction area.

One can simplify Eq. (5) further by assuming that one can approximately express dPDFs as

$$
D_{i, j}\left(x_{i}, x_{j}\right) \approx f_{i}\left(x_{i}\right) f_{j}\left(x_{j}\right),
$$

where $f_{i}$ and $f_{j}$ are standard collinear PDFs. Substituting Eq. (7) in Eq. (5), we arrive to a compact expression for $\sigma_{\mathrm{I}}^{\mathrm{DPS}}$,

$$
\sigma_{\mathrm{I}}^{\mathrm{DPS}}=A \sigma_{\mathrm{pp}}^{\mathrm{DPS}}=\frac{A}{1+\delta_{a b}} \frac{\sigma_{a} \sigma_{b}}{\sigma_{\mathrm{eff}}} .
$$

We see that $\sigma_{\mathrm{I}}^{\text {DPS }}$ scales simply as a total number of nucleons $A$. The DPS II contribution, however, scales differently. The expression for $\sigma_{\text {II }}^{\text {DPS }}$ was found to be equal to 


$$
\begin{aligned}
\sigma_{\mathrm{II}}^{\mathrm{DPS}} & =\frac{1}{1+\delta_{a b}} \frac{A-1}{A} \sigma_{a} \sigma_{b} \int d^{2} s T_{A}^{2}(\mathbf{s}) \\
& =\frac{1}{1+\delta_{a b}} \sigma_{a} \sigma_{b} F_{\mathrm{p} A}
\end{aligned}
$$

where

$$
F_{\mathrm{p} A}=\frac{A-1}{A} \int d^{2} s T_{A}^{2}(\mathbf{s})
$$

and the factor $(A-1) / A$ is the number of possible nucleon pairs $A(A-1)$ divided by $A^{2}$ which comes from normalization of a two nucleon form factor [49], and $T_{A}$ is a nuclear density function $\rho_{A}(r)$ integrated over a longitudinal coordinate

$$
T_{A}(\mathbf{s})=\int d z \rho_{A}(\mathbf{s}, z)
$$

where $\rho_{A}(r)$ obeys a standard normalization condition

$$
\int d^{3} r \rho_{A}(r)=A
$$

Note that only the DPS I contribution depends on $\sigma_{\text {eff }}$ which, in turn, is sensitive to partonic correlations in a transverse plane of a hadron; see [23,50-58] and the review [59].

Combining DPS I and DPS II contributions together, one can write Eq. (1) as

$$
\sigma_{\mathrm{p} A}^{\mathrm{DPS}}=\sigma_{\mathrm{pp}}^{\mathrm{DPS}}\left(A+\sigma_{\mathrm{eff}} F_{\mathrm{p} A}\right) .
$$

We see that within Strikman and Treleani approach one can express the difference between $\sigma_{\mathrm{pp}}^{\mathrm{DPS}}$ and $\sigma_{\mathrm{p} A}^{\mathrm{DPS}}$ solely in terms of a geometrical quantity $T_{A}(\mathbf{s})$ which, in turn, depends on a distribution of matter in a given nucleus. In order to perform numerical evaluations with this formula, one has to specify a form of the nuclear matter density function $\rho_{A}$ which we choose to have a shape of the WoodsSaxon potential [60]

$$
\rho_{A}(r)=\rho_{0} \frac{1+\omega\left(r / R_{A}\right)^{2}}{1+\exp \left[\left(r-R_{A}\right) / a\right]},
$$

where $R_{A}$ is a nuclear radius, $a$ is a length of smearing of a nuclear surface, $\omega$ describes a deviation from a spherical form, and a value of $\rho_{0}$ is fixed by Eq. (12). If one considers a spherical nucleus, the Woods-Saxon nuclear matter density function reduces to the Fermi distribution

$$
\rho_{A}(r)=\frac{\rho_{0}}{1+\exp \left[\left(r-R_{A}\right) / a\right]}
$$

In order to perform numerical evaluations, we need to choose a special parametrization of a nuclear matter density. In this work, we use a parametrization of the GLISSANDO 2 code [61] (the same parametrization as in PYTHIA8). Namely, for nuclei with mass numbers in a range $4 \leq A \leq 208$, we use Wood-Saxon (Fermi) profile given by Eq. (15) with

$$
\begin{gathered}
R_{A}=\left[1.10 A^{1 / 3}-0.656 A^{-1 / 3}\right] \mathrm{fm}, \\
a=0.459 \mathrm{fm},
\end{gathered}
$$

which corresponds to spherical nuclei with a nucleonnucleon $(N N)$ repulsion distance equal to $d=0.9 \mathrm{fm}$.

Now, we can evaluate $A+\sigma_{\mathrm{eff}} F_{\mathrm{p} A}$. In order to estimate the impact due to the uncertainty in the value of $\sigma_{\text {eff }}$, we vary it in between 10 and $20 \mathrm{mb}$ which lies in the range of most experimental studies of four-jet DPS production $[1-3,17]$. In Fig. 2 , we plot the ratio $\sigma_{\mathrm{p} A}^{\mathrm{DPS}} / A \sigma_{\mathrm{pp}}^{\mathrm{DPS}}=1+$ $\frac{1}{A} \sigma_{\mathrm{eff}} F_{\mathrm{pA}}$ as a function of $A$. In the absence of the second term in Eq. (1), this ratio would always be equal to unity. However, we see that a total DPS cross section for heavy nuclei in pA collisions is about $3 A$ times bigger as a corresponding one in pp collisions. We also see that variation of $\sigma_{\text {eff }}$ leads to significant changes in behavior of $\sigma_{\mathrm{p} A}^{\mathrm{DPS}} / A \sigma_{\mathrm{pp}}^{\mathrm{DPS}}$. Such numerical estimate was first made in [22] and the enhancement $\sim 3 A$ was later given in $[23,24,30,31]$. It is handy to approximate a behavior of the DPS enhancement factor $\sigma_{\mathrm{p} A}^{\mathrm{DPS}} / A \sigma_{\mathrm{pp}}^{\mathrm{DPS}}$ as

$$
\frac{1}{A} \frac{\sigma_{\mathrm{p} A}^{\mathrm{DPS}}}{\sigma_{\mathrm{pp}}^{\mathrm{DPS}}}=1+C_{1}(A-1)^{C_{2}}+C_{3}(A-1)^{C_{4}},
$$

where a second term was added to correctly describe enhancement for heavy nuclei and the coefficients $C_{1}-C_{4}$ can be identified by fitting Eq. (18) to our simulations, as it is shown in Fig. 2. This fitting may look somewhat superfluous, since computations within the

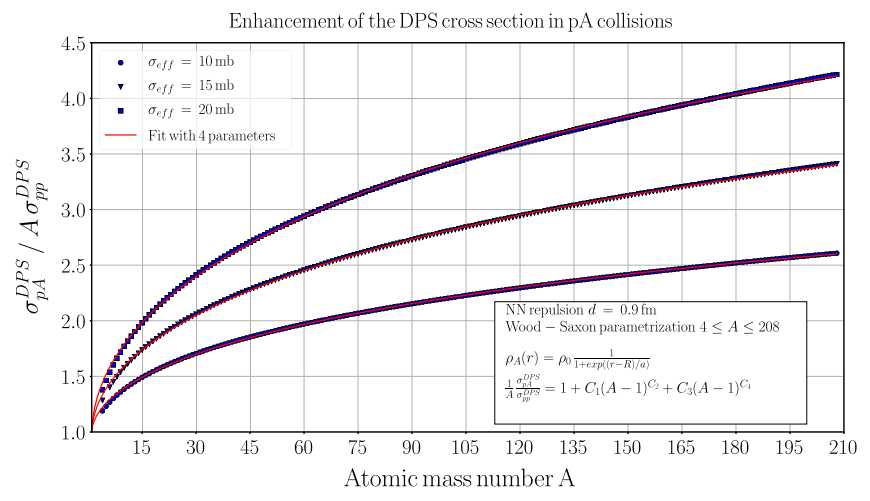

FIG. 2. Enhancement of the $\sigma_{\mathrm{p} A}^{\mathrm{DPS}}$ with respect to $\sigma_{\mathrm{pp}}^{\mathrm{DPS}}$ normalized according to the atomic mass number A. Wood-Saxon (Fermi) form of the nuclear matter distribution $\rho_{A}(r)$ with parameters taken from [61]. 
Strikman and Treleani framework are not time consuming; however, its advantage will become clear later when we will discuss our Monte Carlo simulations.

It is important to note that in general two terms in Eq. (1) correspond to different phase spaces and thus a factorized form of a total DPS cross section given in Eq. (13) violates conservation of a longitudinal momentum. It becomes clear if one writes down expressions for $\sigma_{\mathrm{I}}^{\mathrm{DPS}}$ and $\sigma_{\mathrm{II}}^{\mathrm{DPS}}$,

$$
\begin{aligned}
\sigma_{\mathrm{I}}^{\mathrm{DPS}} & =\frac{1}{1+\delta_{a b}} \frac{A}{\sigma_{\mathrm{eff}}} \sum_{a_{1}, a_{2}, b_{1}, b_{2}} \int \prod_{i=1}^{4} d x_{i} D_{a_{1}, a_{2}}\left(x_{1}, x_{2}\right) D_{b_{1}, b_{2}}\left(x_{3}, x_{4}\right) \hat{\sigma}_{a_{1}, b_{1}} \hat{\sigma}_{a_{2}, b_{2}}, \\
\sigma_{\mathrm{II}}^{\mathrm{DPS}} & =\frac{1}{1+\delta_{a b}} F_{\mathrm{p} A} \sum_{a_{1}, a_{2}, b_{1}, b_{2}} \int \prod_{i=1}^{4} d x_{i} D_{a_{1}, a_{2}}\left(x_{1}, x_{2}\right) f_{b_{1}}\left(x_{3}\right) f_{b_{2}}\left(x_{4}\right) \hat{\sigma}_{a_{1}, b_{1}} \hat{\sigma}_{a_{2}, b_{2}},
\end{aligned}
$$

where, as in Eq. (3), we assume factorization of gPDFs into collinear and transverse pieces and $\sigma_{\text {eff }}$ as given by Eq. (6).

Assuming no correlations in $x$ space, Eqs. (19) and (20) can be written as

$$
\begin{gathered}
\sigma_{\mathrm{I}}^{\mathrm{DPS}}=\frac{1}{1+\delta_{a b}} \frac{A}{\sigma_{\mathrm{eff}}} \sum_{a_{1}, a_{2}, b_{1}, b_{2}} \int \prod_{i=1}^{4} d x_{i} f_{a_{1}}\left(x_{1}\right) f_{a_{2}}\left(x_{2}\right) f_{b_{1}}\left(x_{3}\right) f_{b_{2}}\left(x_{4}\right) \theta\left(1-x_{1}-x_{2}\right) \theta\left(1-x_{3}-x_{4}\right) \hat{\sigma}_{a_{1}, b_{1}} \hat{\sigma}_{a_{2}, b_{2}}, \\
\sigma_{\mathrm{II}}^{\mathrm{DPS}}=\frac{1}{1+\delta_{a b}} F_{\mathrm{p} A} \sum_{a_{1}, a_{2}, b_{1}, b_{2}} \int \prod_{i=1}^{4} d x_{i} f_{a_{1}}\left(x_{1}\right) f_{a_{2}}\left(x_{2}\right) f_{b_{1}}\left(x_{3}\right) f_{b_{2}}\left(x_{4}\right) \theta\left(1-x_{1}-x_{2}\right) \theta\left(x_{3}\right) \theta\left(x_{4}\right) \hat{\sigma}_{a_{1}, b_{1}} \hat{\sigma}_{a_{2}, b_{2}} .
\end{gathered}
$$

We see that different constraints on Bjorken- $x$ 'es lead to different integration regions which does not let us to write a total DPS cross section as in Eq. (13), which in turn means that the value of $\sigma_{\text {eff }}$ being extracted from the data with the help of Eqs. (21) and (22) will differ from the value of $\sigma_{\text {eff }}$ extracted according to Eq. (13). However, the difference between two phase spaces should become relevant only for large $x$ 'es where dPDFs have relatively small values and therefore their impact on a total DPS cross section is small. The direct numerical check gives the difference which is well below the percent level and, therefore, is completely negligible.

\section{ANGANTYR AND MPI MODELS}

Now, let us turn our attention to a Monte Carlo approach to $\mathrm{p} A$ collisions. Usually, in this field, existing Monte Carlo event generators are more "special purpose" and mostly dedicated to studies of formation and evolution of the quark-gluon plasma, e.g., EPOS-LHC [62], AMPT [63], and HIJING [64]. From the other side, there are models postulating flowlike effects to have a nonthermal origin and therefore aiming to reproduce general features of $\mathrm{pA}$ (AA) collisions by adding a nuclear structure "on top" of existing pp models. One such model is called Angantyr [36] that was recently implemented into PYTHIA8 event generator. It was inspired by the old Lund Fritiof model [65] and the DIPSY model [66] [69-71].

The production of final state particles in Angantyr is based upon PYTHIA's models for MPI [72-77] and diffractive processes [78] with certain modifications which will be explained below.

First of all, let us describe the way PYTHIA production of particles in interactions involving one incident proton and a single nucleon as in Fig. 3(a). In this case, one could naively expect that all MPIs would be distributed according to a Poissonian distribution. This approach to MPI modeling, however, may lead to momentum violation and is in contradiction with Koba-Nielsen-Olesen scaling [79] of charged multiplicity distributions; see review [80]. In order to solve this issue, all MPIs in PYTHIA are ordered in transverse momentum as $\sqrt{s} / 2>p_{\perp 1}>p_{\perp 2}>\ldots>p_{\perp n}>p_{\perp \min }$. A probability of a first interaction to happen at a given

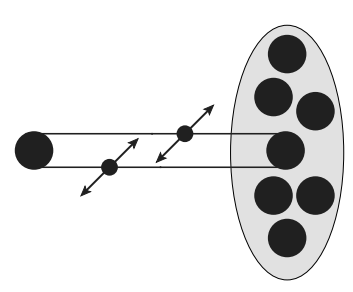

(a)

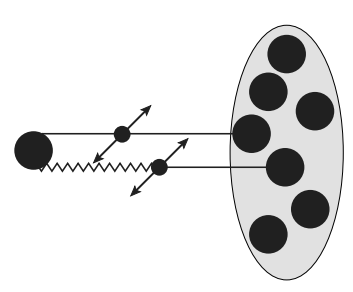

(b)
FIG. 3. A schematic representation of DPS processes in $\mathrm{p} A$ collision according to the Angantyr model. (a) DPS occurs between one incident proton and one nucleon (modeled with a standard MPI machinery). (b) DPS occurs between one incident proton and two different nucleons (modelled with a simplified MPI machinery and modified diffractive machinery). A zigzag line here corresponds to a Pomeron inside of an incident proton. 
transverse momentum $d \sigma / d p_{\perp 1} \sigma_{\mathrm{ND}}^{\mathrm{pp}}(s)$ is multiplied by a Sudakov-like exponent,

$$
\begin{aligned}
\frac{d \mathcal{P}}{d p_{\perp 1}}= & \frac{1}{\sigma_{\mathrm{ND}}^{\mathrm{p}}(s)} \frac{d \sigma}{d p_{\perp 1}} \\
& \times \exp \left(-\int_{p_{\perp 1}}^{\sqrt{s} / 2} \frac{1}{\sigma_{\mathrm{ND}}^{\mathrm{pp}}(s)} \frac{d \sigma}{d p_{\perp}^{\prime}} d p_{\perp}^{\prime}\right),
\end{aligned}
$$

which ensures that no other interactions will happen in $p_{\perp}$ range between $\sqrt{s} / 2$ and $p_{\perp 1}$. Therefore, a probability for all subsequent interactions is given by

$$
\begin{aligned}
\frac{d \mathcal{P}}{d p_{\perp i}}= & \frac{1}{\sigma_{\mathrm{ND}}^{\mathrm{pp}}(s)} \frac{d \sigma}{d p_{\perp i}} \\
& \times \exp \left(-\int_{p_{\perp i}}^{p_{\perp i-1}} \frac{1}{\sigma_{\mathrm{ND}}^{\mathrm{pp}}(s)} \frac{d \sigma}{d p_{\perp}^{\prime}} d p_{\perp}^{\prime}\right)
\end{aligned}
$$

which ensures the $p_{\perp}$ ordering. In addition to it, the MPI model of PYTHIA accounts for momentum and number conservation which implies that PDFs used for a second interaction (as well as for all subsequent interactions) will be "squeezed" and reweighted according to a history of all previous interactions in order to take into account changes in the parton content and preserve momentum conservation; see [74]. It should be noted that $\sigma_{\text {eff }}$ does not enter explicitly into this model. More specifically a ratio $\sigma_{\mathrm{ND}}^{\mathrm{pp}}(s) / \sigma_{\text {eff }}$ describes a deviation of a distribution of MPIs from a Poissonian distribution; see $[74,81]$, and review [80]. However, it implies that direct comparison between predictions of MPI model of PYTHIA and DPS model of Strikman and Treleani is not possible meaning that a corresponding value of parameter $\sigma_{\text {eff }}$ is unknown. We will come back to this issue later in Sec. IV.

Description of processes involving one incoming proton and two different nucleons is somewhat more complicated. In principle, one should have implemented the same contribution as it is shown in Fig. 1(b). However, in practice, incorporation of such processes into PYTHIA's framework leads to serious technical difficulties. It is possible to circumvent these issues by mimicking a second absorptive interaction as in Fig. 1(b) via nucleon-Pomeron collision as in Fig. 3(b). Therefore, in order to simulate double absorptive process from Fig. 1(b), Angantyr will first simulate a single absorptive process via a standard $p p$ machinery and then simulate a second absorptive process as if was produced through a single diffractive excitation, much in the spirit of the old Fritiof model. All subsequent interactions will be produced via standard pp or protonPomeron MPI machinery. Energy-momentum conservation is ensured when extracting the fictitious Promeron from the projectile proton, but this will not influence the PDFs of the proton, and except for the proton remnants the primary absorptive process will look exactly like a normal nondiffractive pp event.

There are several ways to produce diffractive events in PYTHIA8. The Angantyr model is based upon a model of soft diffraction of PYTHIA. For high-mass diffraction, PYTHIA uses the Ingelman and Schlein model where the Pomeron is treated as a hadronic state [82]. Within this approach, PYTHIA treats a proton-Pomeron collision as a normal nondiffractive hadron-hadron collision with standard MPI, initial and final state radiation machinery. Therefore, a corresponding differential $2 \rightarrow 2$ cross section is given by

$$
\begin{aligned}
d \sigma_{i j}^{\mathrm{pP}}= & \frac{d x_{\mathbb{P}}}{x_{\mathbb{P}}} \frac{d x_{1}}{x_{1}} \frac{d \beta}{\beta} \\
& \times F\left(x_{\mathbb{P}}\right) x_{1} f_{i}\left(x_{1}, Q^{2}\right) \beta f_{j / \mathbb{P}}\left(\beta, Q^{2}\right) d \hat{\sigma}_{i j},
\end{aligned}
$$

where $x_{\mathbb{P}}$ is a fraction of the target proton momentum taken by the Pomeron, $\beta$ is a fraction of the Pomeron's momentum taken by the parton $j$, and $x_{1}$ is a fraction of Pomeron's momentum taken by parton $i$. A diffractive mass $M_{X}^{2}$ is therefore given by $M_{X}^{2}=x_{\mathbb{P}} s$. In the Angantyr model, a Pomeron flux $F\left(x_{\mathbb{P}}\right)$ is by default taken to be a constant which implies a flat distribution in $\log \left(M_{X}^{2}\right)$, although this can be changed in the settings. The hard cross section $\hat{\sigma}_{i j}$ in Eq. (25) is the standard leading order (LO) $2 \rightarrow 2$ cross section, which is known to be divergent for low $p_{\perp}$ values. As in the pp case, PYTHIA imposes a smooth cutoff on $\hat{\sigma}_{i j}$ according to

$$
\frac{d \hat{\sigma}_{i j}}{d p_{\perp}^{2}} \propto \frac{\alpha_{s}^{2}\left(p_{\perp}^{2}\right)}{p_{\perp}^{4}} \rightarrow \frac{\alpha_{s}^{2}\left(p_{\perp}^{2}+p_{\perp 0}^{2}\right)}{\left(p_{\perp}^{2}+p_{\perp 0}^{2}\right)^{2}}
$$

where $p_{\perp 0}$ is a soft regulator which depends either on diffractive mass (for diffractive processes) or on collision energy (for standard pp processes). Nevertheless, even after a regularization of $\hat{\sigma}_{i j}$ as in Eq. (26), an integrated partonic cross section may exceed a total nondiffractive protonPomeron cross section for a given diffractive mass $M_{X}$. In the MPI model of PYTHIA, it is interpreted as a possibility to have several subscatterings in each collision with an average number,

$$
\left\langle N_{s c}^{\mathrm{p} \mathbb{P}}\left(M_{X}^{2}\right)\right\rangle=\frac{1}{\sigma_{\mathrm{ND}}^{\mathrm{pP}}\left(M_{X}\right)} \int \frac{d x_{1}}{x_{1}} \frac{d \beta}{\beta} d p_{\perp}^{2} \sum_{i j} x_{1} f_{i}\left(x_{1}, Q^{2}\right) \beta f_{j / \mathbb{P}}\left(\beta, Q^{2}\right) \frac{d \hat{\sigma}_{i j}}{d p_{\perp}^{2}} .
$$


However, as it was pointed out in [83], a modeling of single absorptive events via single diffractive (SD) events results in too low activity in $\mathrm{p} A$ collision. In principle, one can solve this problem either by tuning the value of $\sigma_{\mathrm{ND}}^{\mathrm{pP}}\left(M_{X}\right)$ in Eq. (27) or by changing Pomeron PDFs. By comparing a distribution $d\left\langle N_{s c}^{\mathrm{pP}}\right\rangle / d y$ for SD events

$$
\frac{d\left\langle N_{s c}^{\mathrm{p} \mathbb{P}}\right\rangle}{d y}=\frac{1}{\sigma_{\mathrm{ND}}^{\mathrm{p}}\left(M_{X}^{2}\right)} \int \frac{d x_{1}}{x_{1}} \frac{d \beta}{\beta} d p_{\perp}^{2} \sum_{i j} x_{1} f_{i}\left(x_{1}, Q^{2}\right) \beta f_{j / \mathbb{P}}\left(\beta, Q^{2}\right) \frac{d \hat{\sigma}_{i j}}{d p_{\perp}^{2}} \delta\left(y-\frac{1}{2} \log \frac{x_{1}}{\beta x_{\mathbb{P}}}\right)
$$

against a corresponding distribution for standard nondiffractive pp events

$$
\frac{d\left\langle N_{s c}^{\mathrm{pp}}\right\rangle}{d y}=\frac{1}{\sigma_{\mathrm{ND}}^{\mathrm{pp}}(s)} \int \frac{d x_{1}}{x_{1}} \frac{d x_{2}}{x_{2}} d p_{\perp}^{2} \sum_{i j} x_{1} f_{i}\left(x_{1}, Q^{2}\right) x_{2} f_{j}\left(x_{2}, Q^{2}\right) \frac{d \hat{\sigma}_{i j}}{d p_{\perp}^{2}} \delta\left(y-\frac{1}{2} \log \frac{x_{1}}{x_{2}}\right),
$$

we see that if in Eq. (28) we set $\beta f_{j / \mathbb{P}}\left(\beta, Q^{2}\right) \rightarrow$ $x_{\mathbb{P}} \beta f_{j}\left(x_{\mathbb{P}} \beta, Q^{2}\right), \sigma_{\mathrm{ND}}^{\mathrm{pP}}\left(M_{X}\right) \rightarrow \sigma_{\mathrm{ND}}^{\mathrm{pp}}(s)$, then we get an expression very similar to Eq. (29). Also, if the energy dependence of soft regularization in $\hat{\sigma}$ is changed from $p_{\perp 0}\left(M_{X}^{2}\right)$ to $p_{\perp 0}(s)$, the expression will be identical for large negative rapidities, which is what is desired.

The validity of this approach was studied in detail in [36]. In particular, it was shown that Eq. (28), modified as described, provides an overall fair description of experimental data. However, all Angantyr checks in [36] were related to MPI-sensitive distributions like, for example, a charged multiplicity distribution. Indeed, such distributions are known to be very sensitive to a number of semihard and soft subcollisions in a given event; see, for example, review [80]. Therefore, correct predictions for a shape of such distributions can be seen as a validation of both MPI and Angantyr models. In the next section of this paper, we will switch our attention from MPI to DPS processes and perform another check of the Angantyr model. Namely, we will study how well it can reproduce predictions of Strikman and Treleani for DPS production of four hard jets in $\mathrm{p} A$ collisions.

\section{PREDICTIONS OF PYTHIA}

Before starting to compare predictions of PYTHIA against Strikman and Treleani model, several important comments have to be made. First of all, as we already mentioned in Sec. III, all MPIs produced in a given event are strictly ordered in $p_{\perp}$. This ordering may seem to be in contradiction with the Strikman and Treleani model, where the two processes are treated equal. However, here we will only consider the case of having two identical processes, so this is then just a trivial numbering issue. One should also keep in mind that, in order to derive Eq. (13), Strikman and Treleani assumed that both DPS I and DPS II contributions populate the same phase space region which essentially implies the absence of $\theta$ functions constrains in Eqs. (21) and (22). However, as we have noticed in Sec. II, the error due to this approximation is completely negligible. We also shall notice that both PYTHIA and Strikman and Treleani models do not take partonic correlations (e.g., in spin, color) into account. The incorporation of such effects in the DPS framework is a highly nontrivial task $[44,45]$ and is beyond the scope of this paper. Nevertheless, we should keep in mind that PYTHIA's approach to momentum and number conservation effectively means the presence of nontrivial $x$-space partonic correlations in the MPI machinery and that correlations in $x$ space, among other possible correlations, may have a sizeable impact on the DPS processes; see [24] and [84-86].

Finally, we need to stress that the parameter $\sigma_{\text {eff }}$ does not enter explicitly into the Angantyr model and, therefore, in order to compare the predictions of Strikman and Treleani model against predictions of Angantyr, one has to find the value of $\sigma_{\text {eff }}$ in Strikman and Treleani model by fitting its predictions to the prediction of Angantyr.

Now, after describing all the important differences between both approaches, let us study how the DPS enhancement factor $\sigma_{\mathrm{p} A}^{\mathrm{DPS}} / A \sigma_{\mathrm{pp}}^{\mathrm{DPS}}$ in the Angantyr model depends on a total number of nucleons $A$.

Due to the lack of triggering in the MPI machinery, one will need to perform a high number of generation calls in order to collect a good statistics for a four-jet DPS production, since a second MPI will most of the time occur at too low scale to be considered as a hard interaction [87]. Therefore, we evaluate $\sigma_{\mathrm{p} A}^{\mathrm{DPS}}$ according to following algorithm:

(i) Find a total weight $w_{\mathrm{pA}}^{\text {tot }}$ for all events produced in $\mathrm{p} A$ collisions and a corresponding total cross section $\sigma_{\mathrm{p} A}^{\mathrm{tot}}$.

(ii) Find a total weight $w_{\mathrm{p} A}^{\mathrm{DPS}}$ of all events which satisfy a given set of cuts.

(iii) Find a total DPS cross section in $\mathrm{p} A$ collisions $\sigma_{\mathrm{p} A}^{\mathrm{DPS}}$ from the ratio

$$
\frac{\sigma_{\mathrm{p} A}^{\mathrm{DPS}}}{\sigma_{\mathrm{p} A}^{\text {tot }}}=\frac{w_{\mathrm{p} A}^{\mathrm{DPS}}}{w_{\mathrm{p} A}^{\text {tot }}} .
$$

(iv) Repeat the same for pp collisions. Find a corresponding total DPS cross section $\sigma_{\mathrm{pp}}^{\mathrm{DPS}}$.

(v) Evaluate $\sigma_{\mathrm{p} A}^{\mathrm{DPS}} / A \sigma_{\mathrm{pp}}^{\mathrm{DPS}}$. 
TABLE I. PYTHIA: predictions for enhancement factor for DPS in $\mathrm{p} A$ collisions at $\sqrt{S_{N N}}=5 \mathrm{TeV}\left(10^{7}\right.$ PYTHIA calls $)$.

\begin{tabular}{lcc}
\hline \hline Nucleus & Angantyr SDTries $=1$ & Angantyr SDTries $=2$ \\
\hline${ }^{4} \mathrm{He}$ & 1.12 & 1.12 \\
${ }^{6} \mathrm{Li}$ & 1.18 & 1.18 \\
${ }^{12} \mathrm{C}$ & 1.34 & 1.36 \\
${ }^{16} \mathrm{O}$ & 1.43 & 1.44 \\
${ }^{63} \mathrm{Cu}$ & 2.03 & 2.03 \\
${ }^{129} \mathrm{Xe}$ & 2.46 & 2.49 \\
${ }^{197} \mathrm{Au}$ & 2.80 & 2.80 \\
${ }^{208} \mathrm{~Pb}$ & 2.82 & 2.84 \\
\hline \hline
\end{tabular}

In principle, $\mathrm{p} A$ machinery of PYTHIA allows a user to implement any isotop with given values of $Z$ and $N$. Eight nuclei: ${ }^{4} \mathrm{He},{ }^{6} \mathrm{Li},{ }^{12} \mathrm{C},{ }^{16} \mathrm{O},{ }^{63} \mathrm{Cu},{ }^{129} \mathrm{Xe},{ }^{197} \mathrm{Au}$, and ${ }^{208} \mathrm{~Pb}$ are available by default. Since a computation of a total DPS cross section according to the algorithm above can take tens of hours (depending on a chosen nucleus and a system performance), we decided to work only with already implemented nuclei and use a fit as in Eq. (18) for better visualization of our results and for comparison against Strikman and Treleani model.

Our results for $\sigma_{\mathrm{p} A}^{\mathrm{DPS}} / A \sigma_{\mathrm{pp}}^{\mathrm{DPS}}$ are given in Table I. In our simulations, we were triggering on events with at least four jets with $p_{\perp}>20 \mathrm{GeV}$. We have also performed a stability check by varying a parameter Angantyr:SDTries controlling the maximum number of attempts allowed to add a secondary absorptive subevent [as in Fig. 3(b)] without violating energy-momentum conservation. By comparing values of $\sigma_{\mathrm{p} A}^{\mathrm{DPS}} / A \sigma_{\mathrm{pp}}^{\mathrm{DPS}}$ evaluated at different values of SDTries parameter, we see that fluctuations of $\sigma_{\mathrm{p} A}^{\mathrm{DPS}} / A \sigma_{\mathrm{pp}}^{\mathrm{DPS}}$ do not exceed a few percent level.

A comparison against Strikman and Treleani model is given in Fig. 4 and Table II. The PYTHIA setup we use is given in Table III. In order to compare our results against Strikman and Treleani model, we have tuned $\sigma_{\text {eff }}$ in order to

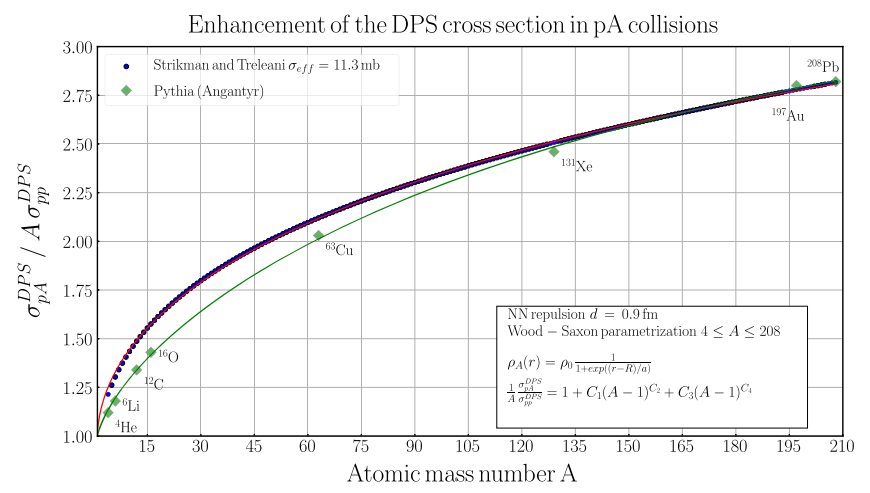

FIG. 4. The DPS enhancement factor $\sigma_{\mathrm{p} A}^{\mathrm{DPS}} / A \sigma_{\mathrm{pp}}^{\mathrm{DPS}}$ as a function of a total number of nucleons $A$. Comparison between theoretical predictions of Strikman and Treleani [22] and PYTHIA's (Angantyr) simulations.
TABLE II. PYTHIA: predictions for enhancement factor $\sigma_{\mathrm{p} A}^{\mathrm{DPS}} / A \sigma_{\mathrm{pp}}^{\mathrm{DPS}}$ at $\sqrt{S_{N N}}=5 \mathrm{TeV}\left(10^{7}\right.$ PYTHIA calls $)$. In Strikman and Treleani model, we set $\sigma_{\text {eff }}=11.3 \mathrm{mb}$.

\begin{tabular}{lcc}
\hline \hline Nucleus & Angantyr SDTries $=1$ & Strikman and Treleani \\
\hline${ }^{4} \mathrm{He}$ & 1.12 & 1.21 \\
${ }^{6} \mathrm{Li}$ & 1.18 & 1.30 \\
${ }^{12} \mathrm{C}$ & 1.34 & 1.49 \\
${ }^{16} \mathrm{O}$ & 1.43 & 1.58 \\
${ }^{63} \mathrm{Cu}$ & 2.03 & 2.12 \\
${ }^{129} \mathrm{Xe}$ & 2.46 & 2.51 \\
${ }^{197} \mathrm{Au}$ & 2.80 & 2.78 \\
${ }^{208} \mathrm{~Pb}$ & 2.82 & 2.82 \\
\hline \hline
\end{tabular}

get an agreement in the value of the DPS enhancement factor $\sigma_{\mathrm{p} A}^{\mathrm{DPS}} / A \sigma_{\mathrm{pp}}^{\mathrm{DPS}}$ for ${ }^{208} \mathrm{~Pb}$. We see that by choosing $\sigma_{\text {eff }}=11.3 \mathrm{mb}$ we can get a satisfactory agreement between both models for heavy isotopes ${ }^{129} \mathrm{Xe},{ }^{197} \mathrm{Au}$, and ${ }^{208} \mathrm{~Pb}$.

It could be tempting to interpret our simulations as a fake data and to use Eq. (13) for a fitting procedure to extract a value of $\sigma_{\text {eff }}$ out of it. However, due to the differences between the models, such an interpretation would not be very relevant. As we have mentioned at the end of Sec. II, the constraints due to the longitudinal momentum conservation do not allow us to express $\sigma_{\mathrm{p} A}^{\mathrm{DPS}}$ as in Eq. (13). Speaking in more general terms, any model of DPS in $\mathrm{pA}$ which accounts for partonic correlations or DPS processes different from those shown in Fig. 1, being applied to the experimental data may give the values of $\sigma_{\text {eff }}$ different from those obtained with Eq. (13). For example, as it was shown

TABLE III. The PYTHIA settings used for the presented predictions.

\begin{tabular}{lc}
\hline \hline PYTHIA settings & Value \\
\hline Random:setSeed & On \\
HardQCD:all & On \\
PartonLevel:mpi & On \\
PartonLevel:remnants & On \\
Check:event & On \\
PartonLevel:isr & Off \\
PartonLevel:fsr & Off \\
ColourReconnection:reconnect & Off \\
HadronLevel:all & Off \\
Beams:idA & 2212 \\
Beams:idB & 1000020040 (as an \\
& example for $\left.{ }^{4} \mathrm{He}\right)$ \\
Beams:eA & $4000 \mathrm{GeV}$ \\
Beams:eB & $1570 \mathrm{GeV}$ \\
Beams:frameType & 2 \\
PDF:pSet & LHAPDF6:MSTW2008lo68cl \\
PhaseSpace:pTHatMin & $20.0 \mathrm{GeV}$ \\
SigmaProcess:renormScale2 & 2 \\
SigmaProcess:factorScale2 & 2 \\
\hline \hline
\end{tabular}




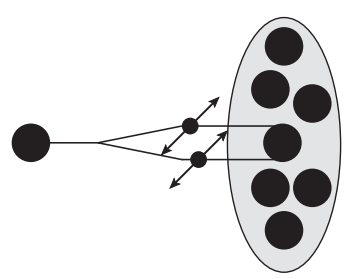

(a)

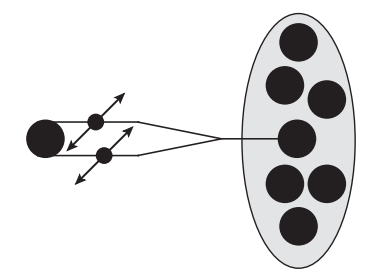

(b)

FIG. 5. A schematic representation of some possible " $1 v 2$ " DPS processes in $\mathrm{p} A$ collision. (a) A $1 v 2$ splitting occurs in the incident proton before two hard interactions take place. (b) A $1 v 2$ splitting occurs in a nucleon before two hard interactions take place.

in [24], perturbative splittings of initial state partons lead to additional DPS contributions which have different importance for different nuclei. Whereas the MPI model of PYTHIA partially accounts [88] for processes shown in Fig. 5, incorporation of such terms in the Strikman and Treleani framework is a nontrivial task; see $[89,90]$. Nevertheless, we would like to stress that the enhancement $\sim 3 A$ of a DPS cross section in $\mathrm{p} A$ collisions is a prediction based solely on geometry of a composite nuclear target and, therefore, any model of DPS in $\mathrm{p} A$ collision should demonstrate a behavior of the DPS enhancement factor $\sigma_{\mathrm{p} A}^{\mathrm{DPS}} / A \sigma_{\mathrm{pp}}^{\mathrm{DPS}}$ similar to the one shown in Fig. 2. In this sense, the comparison of the predictions of Angantyr against predictions made with Eq. (13) is correct to a first approximation.

Before finishing this section, we would like to give an example how the differences between Angantyr and Strikman and Treleani models discussed above can be found in the data. In the previous simulations, we triggered on events with at least four jets with $p_{\perp}>20 \mathrm{GeV}$ without imposing any cuts on their rapidities. However, it is known that activity in $\mathrm{p} A$ collisions depends on rapidity of produced particles in a nontrivial way. Namely, as it was observed by the first time by Busza et al. [91], the charged multiplicity distribution $d N_{c h} / d \eta$ in $\mathrm{p} A$ collisions grows for the negative values of $\eta$ (assuming that the nucleus $A$ is located in the negative direction of the $\eta$ axis). There are several explanation of this phenomenon. For example, it can be explained by a Balitsky-Fadin-Kuraev-Lipatov (BFKL) evolution of a gluon cascade in rapidity-impactparameter space, as it is sketched in Fig. 6, where a probability to have several absorptive interactions grows

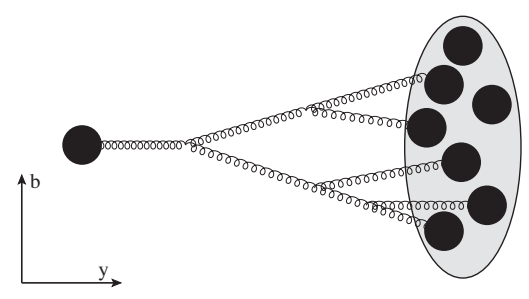

FIG. 6. A schematic representation of a BFKL evolution of a gluon cascade in rapidity-impact-parameter space.

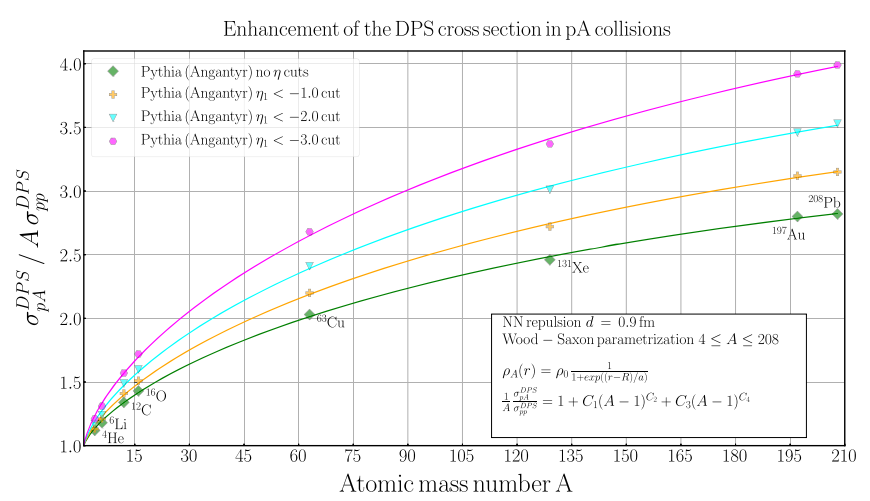

FIG. 7. Dependence of the enhancement factor $\sigma_{\mathrm{p} A}^{\mathrm{DPS}} / A \sigma_{\mathrm{pp}}^{\mathrm{DPS}}$ on $\eta$ cuts. Predictions of PYTHIA (Angantyr). Here orange, cyan, and magenta curves correspond to four-jet DPS production with at least one jet with $\eta$ smaller than $-1,-2$, and -3 correspondingly.

in a direction of a nucleus. The same result can be explained by the original nonperturbative "wounded nucleon model" [92-94] (which is also the basis of the Fritiof program). These effects are also implemented in the Angantyr model of $\mathrm{p} A$ collisions which, to some extent, can be seen as a perturbative version of the wounded nucleon model which includes MPIs produced according to Eq. (28) with modifications described in Sec. III. As shown in [36], the Angantyr model correctly describes the skewed pseudorapidity distribution. Since the particle production in Angantyr relies on the MPI model of the PYTHIA event generator, the charged multiplicity distribution should be correlated with production of (mini-)jets. More precisely, the growth of charged multiplicity $d N_{c h} / d \eta$ for negative $\eta$ values in Angantyr model is inextricably connected with growth of a number of subscatterings in a given event; see Eqs. (28) and (29). Therefore, it is natural to assume that in the Angantyr model probability to generate an event of a DPS II type will depend on $\eta$ in a way similar to a $d N_{c h} / d \eta$ distribution. In order to check this, we evaluate $\sigma_{\mathrm{p} A}^{\mathrm{DPS}} / A \sigma_{\mathrm{pp}}^{\mathrm{DPS}}$ for events with at least four jets with $p_{\perp}>20 \mathrm{GeV}$ and at least one jet with a pseudorapidity value smaller [95] than a certain value $\eta_{\text {cut }}$. Obviously, additional $\eta$ cuts will reduce the total DPS cross section in $\mathrm{pp}$ and $\mathrm{pA}$ collisions. Nevertheless, one could expect that the total DPS cross section in the $\mathrm{p} A$ case will decrease much slower than corresponding one in the pp case. As a consequence, the enhancement factor $\sigma_{\mathrm{pA}}^{\mathrm{DPS}} / A \sigma_{\mathrm{pp}}^{\mathrm{DPS}}$ will grow since in the Angantyr model probability to generate a process of DPS II increases at small negative values of $\eta$.

The results are presented in Fig. 7. In order to study how the DPS enhancement factor $\sigma_{\mathrm{p} A}^{\mathrm{DPS}} / A \sigma_{\mathrm{pp}}^{\mathrm{DPS}}$ depends on rapidity cuts, we have used the same setup as before but with additional cuts $\eta_{\text {cut }}=-1, \eta_{\text {cut }}=-2$, and $\eta_{\text {cut }}=-3$. We see that indeed the ratio $\sigma_{\mathrm{p} A}^{\mathrm{DPS}} / A \sigma_{\mathrm{pp}}^{\mathrm{DPS}}$ demonstrates a strong dependence on the value of $\eta_{\text {cut }}$. The experimental verification of the growth of the DPS enhancement factor 
$\sigma_{\mathrm{p} A}^{\mathrm{DPS}} / A \sigma_{\mathrm{pp}}^{\mathrm{DPS}}$ due to the additional rapidity cut predicted by the Angantyr model could, in principle, provide a better way to control the fraction of double absorptive processes shown in Fig. 3(b).

\section{CONCLUSIONS}

We have demonstrated that the Angantyr model of $\mathrm{p} A$ collisions in PYTHIA8 predicts an $A$ dependence of a DPS enhancement factor $\sigma_{\mathrm{p} A}^{\mathrm{DPS}} / A \sigma_{\mathrm{pp}}^{\mathrm{DPS}}$ which agrees with the one predicted in a pioneering work of Strikman and Treleani [22] at a qualitative level. This result can be seen as an additional validation of the Angantyr's approach to double absorptive processes described in Sec. III. From the other side, a correct $A$ dependence means that, apart from standard applications, one can use Angantyr for standalone studies of DPS in $\mathrm{p} A$ collisions. In this case, a potential user can benefit not only from evaluation of a total cross section, but also from the most of entire PYTHIA machinery like initial and final state radiation, color reconnections, etc. Furthermore, the availability of a full event generator will allow for a realistic estimate of the effects of the underlying event and other issues associated with the experimental measurements of jets.

We also have studied how (pseudo) rapidity cuts affect the number of MPIs in a given event and therefore a behavior of $\sigma_{\mathrm{p} A}^{\mathrm{DPS}} / A \sigma_{\mathrm{pp}}^{\mathrm{DPS}}$. The growth of $\sigma_{\mathrm{p} A}^{\mathrm{DPS}} / A \sigma_{\mathrm{pp}}^{\mathrm{DPS}}$ is a natural consequence of (pseudo)rapidity dependence of activity in $\mathrm{p} A$ collisions built into the Angantyr model. This behavior was inspired by a DIPSY model and is essential to get a qualitative agreement with available experimental data on $\mathrm{p} A$ collisions; see [36].

One has to keep in mind that the Angantyr model (as well as the MPI model of PYTHIA) is using the assumption about factorization of gPDFs into longitudinally and transversedependent pieces as in Eq. (4). This approximation leads to the effective DPS interaction area defined as in Eq. (6) where integration runs over all possible values of the separation between partons $|\boldsymbol{b}|$. However, as it was discussed in a series of works $[58,89,96-100]$, the integration over all possible values of $|\boldsymbol{b}|$ leads to the overlap between regions of validity of the double and single parton scattering approximation of the hard processes. A consistent scheme that allows to solve this problem in all orders in perturbation theory was proposed in [89], and its first successful application to the same-sign $W$-boson production was recently reported [101]. However, the application of the scheme of [89] to the case of four-jet DPS production requires four-jet single parton scattering cross section at next-to-next-to-leading order accuracy, which is not available at the moment and, therefore, in case of the four-jet DPS production one still has to rely on factorization of gPDFs as in Eq. (4).

Finally, we would like to note that the complexity of the problem of DPS in $\mathrm{p} A$ collisions requires a detailed study of various nontrivial effects, such as partonic correlations, cold nuclear matter effects, and additional DPS contributions, as was pointed out in [24]. Therefore, in the absence of experimental studies of DPS in $\mathrm{p} A$ collisions, a comparison between predictions of Angantyr and improved Strikman and Treleani model may help us to identify key ingredients essential for correct modeling of DPS in $\mathrm{pA}$ collisions. Recently, the improved model of Strikman and Treleani was proposed by Alvioli et al. [27]. In particular, it accounts for color fluctuation effects and allows to compute the DPS cross section as a function of centrality. The latter is crucial for the experimental studies of the DPS phenomena in $\mathrm{p} A$ collisions. Therefore, we argue that, in the absence of experimental measurements of DPS in $\mathrm{p} A$ collisions, the detailed comparison between Angantyr's predictions can be beneficial for better understanding of the DPS phenomena in $\mathrm{p} A$ collisions.

\section{ACKNOWLEDGMENTS}

The work of L. L. was funded in part by the Knut and Alice Wallenberg foundation, Contract No. 2017.0036 and by the Swedish Research Council, Contracts No. 2016-03291 and No. 2016-05996. The work of O. F. has received funding from the European Union's Horizon 2020 research and innovation programme as part of the Marie Skłodowska-Curie Innovative Training Network MCnetITN3 (Grant No. 722104) and partially by the Deutsche Forschungsgemeinschaft through the Research Training Group "GRK 2149: Strong and Weak Interactions - from Hadrons to Dark Matter," by the curiositydriven grant "Using jets to challenge the Standard Model of particle physics" from Università di Genova. O. F. thanks all members of the theoretical particle physics group of the Lund University for warm hospitality and friendly atmosphere, and in particular acknowledges Christian Bierlich, Johannes Bellm, Gösta Gustafson, Anna Kulesza, Harsh Shah, and Torbjörn Sjöstrand for useful and fruitful discussions. All diagrams in this paper were created with the JaxoDraw code [102]. In order to perform numerical computations within Strikman and Treleani framework, we used numerical integration routines from GSL library [103], in particular those based upon VEGAS algorithm by Lepage [104]. All figures in this paper were created with the Matplotlib library [105]. For the fitting purposes, SciPy [106] and NumPy [107] libraries were used. For our computations and simulations involving PDFs, we used LHAPDF6 library [108] and a central value of MSTW2008 LO PDF set [109]. 
[1] T. Åkesson et al. (Axial Field Spectrometer), Z. Phys. C 34, 163 (1987).

[2] J. Alitti et al. (UA2 Collaboration), Phys. Lett. B 268, 145 (1991).

[3] F. Abe et al. (CDF Collaboration), Phys. Rev. D 47, 4857 (1993).

[4] F. Abe et al. (CDF Collaboration), Phys. Rev. Lett. 79, 584 (1997).

[5] F. Abe et al. (CDF Collaboration), Phys. Rev. D 56, 3811 (1997).

[6] V. M. Abazov et al. (D0 Collaboration), Phys. Rev. D 81, 052012 (2010).

[7] M. Aaboud et al. (ATLAS Collaboration), Eur. Phys. J. C 77, 76 (2017).

[8] R. Aaij et al. (LHCb Collaboration), J. High Energy Phys. 06 (2012) 141; 03 (2014) 108(A).

[9] G. Aad et al. (ATLAS Collaboration), New J. Phys. 15, 033038 (2013).

[10] S. Chatrchyan et al. (CMS Collaboration), J. High Energy Phys. 03 (2014) 032.

[11] V. M. Abazov et al. (D0 Collaboration), Phys. Rev. D 90, 111101 (2014).

[12] V. M. Abazov et al. (D0 Collaboration), Phys. Rev. D 89, 072006 (2014).

[13] G. Aad et al. (ATLAS Collaboration), Eur. Phys. J. C 75, 229 (2015).

[14] R. Aaij et al. (LHCb Collaboration), J. High Energy Phys. 07 (2016) 052.

[15] V. M. Abazov et al. (D0 Collaboration), Phys. Rev. Lett. 116, 082002 (2016).

[16] V. M. Abazov et al. (D0 Collaboration), Phys. Rev. D 93, 052008 (2016).

[17] M. Aaboud et al. (ATLAS Collaboration), J. High Energy Phys. 11 (2016) 110.

[18] R. Aaij et al. (LHCb Collaboration), J. High Energy Phys. 06 (2017) 047; 10 (2017) 068(E).

[19] V. Khachatryan et al. (CMS Collaboration), J. High Energy Phys. 05 (2017) 013.

[20] M. Aaboud et al. (ATLAS Collaboration), Phys. Lett. B 790, 595 (2019); 790, 595 (2019).

[21] A. M. Sirunyan et al. (CMS Collaboration), J. High Energy Phys. 02 (2018) 032.

[22] M. Strikman and D. Treleani, Phys. Rev. Lett. 88, 031801 (2002).

[23] L. Frankfurt, M. Strikman, and C. Weiss, Ann. Phys. (Amsterdam) 13, 665 (2004).

[24] B. Blok, M. Strikman, and U. A. Wiedemann, Eur. Phys. J. C 73, 2433 (2013).

[25] M. Strikman and W. Vogelsang, Phys. Rev. D 83, 034029 (2011).

[26] B. Blok and M. Strikman, Adv. Ser. Dir. High Energy Phys. 29, 63 (2018).

[27] M. Alvioli, M. Azarkin, B. Blok, and M. Strikman, Eur. Phys. J. C 79, 482 (2019).

[28] D. d'Enterria and A. M. Snigirev, Phys. Lett. B 727, 157 (2013).

[29] S. Salvini, D. Treleani, and G. Calucci, Phys. Rev. D 89, 016020 (2014).

[30] E. Cattaruzza, A. Del Fabbro, and D. Treleani, Phys. Rev. D 70, 034022 (2004).
[31] D. d'Enterria and A. M. Snigirev, Phys. Lett. B 718, 1395 (2013).

[32] E. R. Cazaroto, V. P. Goncalves, and F. S. Navarra, Phys. Rev. D 88, 034005 (2013).

[33] D. d'Enterria and A. M. Snigirev, Nucl. Phys. A931, 303 (2014).

[34] I. Helenius and H. Paukkunen, Phys. Lett. B 800, 135084 (2020).

[35] B. Blok and F. A. Ceccopieri, Eur. Phys. J. C 80, 278 (2020).

[36] C. Bierlich, G. Gustafson, L. Lönnblad, and H. Shah, J. High Energy Phys. 10 (2018) 134.

[37] T. Sjöstrand, S. Mrenna, and P. Z. Skands, J. High Energy Phys. 05 (2006) 026.

[38] T. Sjöstrand, S. Mrenna, and P. Z. Skands, Comput. Phys. Commun. 178, 852 (2008).

[39] To the best of our knowledge, a similar but somewhat different assumption was first made by Goebel et al. in 1980 [40]. Namely, it was postulated that total cross sections for DPS and standard single parton scattering processes in $\mathrm{p} A$ collision will have a different $A$ dependence. However, no distinction between DPS I and DPS II contributions was made and corresponding expressions for total cross sections were not provided.

[40] C. Goebel, F. Halzen, and D. M. Scott, Phys. Rev. D 22, 2789 (1980).

[41] For the parton model derivation of the expression for the total DPS cross section, see [42-45] and for the full QCD derivation, see [44-48].

[42] N. Paver and D. Treleani, Nuovo Cimento A 70, 215 (1982).

[43] M. Mekhfi, Phys. Rev. D 32, 2371 (1985).

[44] M. Diehl and A. Schafer, Phys. Lett. B 698, 389 (2011).

[45] M. Diehl, D. Ostermeier, and A. Schafer, J. High Energy Phys. 03 (2012) 089; 03 (2016) 001(E).

[46] M. Diehl, J. R. Gaunt, D. Ostermeier, P. Plößl, and A. Schäfer, J. High Energy Phys. 01 (2016) 076.

[47] M. Diehl and R. Nagar, J. High Energy Phys. 04 (2019) 124.

[48] A. Vladimirov, J. High Energy Phys. 04 (2018) 045.

[49] The factor $(A-1) / A$ was absent in the original publication of Strikman and Treleani [22]. It was also absent in a work of Cattaruzza et al. [30] which followed [22]. To the best of our knowledge, this factor appears first in the work of Frankfurt et al. [23], and a detailed derivation was later given in the work of Blok et al. [24].

[50] G. Calucci and D. Treleani, Phys. Rev. D 57, 503 (1998).

[51] L. Frankfurt, M. Strikman, and C. Weiss, Phys. Rev. D 69, 114010 (2004).

[52] G. Calucci and D. Treleani, Phys. Rev. D 60, 054023 (1999).

[53] G. Calucci and D. Treleani, Phys. Rev. D 79, 074013 (2009).

[54] G. Calucci and D. Treleani, Phys. Rev. D 83, 016012 (2011).

[55] T. C. Rogers and M. Strikman, Phys. Rev. D 81, 016013 (2010).

[56] S. Domdey, H.-J. Pirner, and U. A. Wiedemann, Eur. Phys. J. C 65, 153 (2010). 
[57] C. Flensburg, G. Gustafson, L. Lonnblad, and A. Ster, J. High Energy Phys. 06 (2011) 066.

[58] B. Blok, Yu. Dokshitzer, L. Frankfurt, and M. Strikman, Eur. Phys. J. C 74, 2926 (2014).

[59] M. Diehl, Proc. Sci., DIS2013 (2013) 074.

[60] R. D. Woods and D. S. Saxon, Phys. Rev. 95, 577 (1954).

[61] M. Rybczynski, G. Stefanek, W. Broniowski, and P. Bozek, Comput. Phys. Commun. 185, 1759 (2014).

[62] T. Pierog, I. Karpenko, J. M. Katzy, E. Yatsenko, and K. Werner, Phys. Rev. C 92, 034906 (2015).

[63] Z.-W. Lin, C. M. Ko, B.-A. Li, B. Zhang, and S. Pal, Phys. Rev. C 72, 064901 (2005).

[64] X.-N. Wang and M. Gyulassy, Phys. Rev. D 44, 3501 (1991).

[65] B. Andersson, G. Gustafson, and B. Nilsson-Almqvist, Nucl. Phys. B281, 289 (1987).

[66] DIPSY models BFKL evolution of a gluon cascade via Mueller dipole approach $[67,68]$.

[67] A. H. Mueller, Nucl. Phys. B415, 373 (1994).

[68] A. H. Mueller and B. Patel, Nucl. Phys. B425, 471 (1994).

[69] E. Avsar, G. Gustafson, and L. Lonnblad, J. High Energy Phys. 01 (2007) 012.

[70] E. Avsar, G. Gustafson, and L. Lonnblad, J. High Energy Phys. 07 (2005) 062.

[71] C. Flensburg, G. Gustafson, and L. Lonnblad, J. High Energy Phys. 08 (2011) 103.

[72] T. Sjöstrand, Multiple Parton-Parton Interactions in Hadronic Event, in Proceedings, 23RD International Conference on High Energy Physics, Berkeley, CA, July 16-23, 1986 (World Scientific, Singapore, 1987).

[73] T. Sjöstrand and M. van Zijl, Phys. Rev. D 36, 2019 (1987).

[74] T. Sjöstrand and P.Z. Skands, J. High Energy Phys. 03 (2004) 053.

[75] R. Corke and T. Sjöstrand, J. High Energy Phys. 01 (2010) 035.

[76] R. Corke and T. Sjöstrand, J. High Energy Phys. 05 (2011) 009.

[77] R. Corke and T. Sjöstrand, J. High Energy Phys. 03 (2011) 032.

[78] S. Navin, arXiv:1005.3894.

[79] Z. Koba, H. B. Nielsen, and P. Olesen, Nucl. Phys. B40, 317 (1972).

[80] T. Sjöstrand, Adv. Ser. Dir. High Energy Phys. 29, 191 (2018).

[81] M. H. Seymour and A. Siodmok, arXiv:1308.6749.

[82] G. Ingelman and P. E. Schlein, Phys. Lett. 152B, 256 (1985).

[83] C. Bierlich, G. Gustafson, and L. Lönnblad, J. High Energy Phys. 10 (2016) 139.
[84] V. L. Korotkikh and A. M. Snigirev, Phys. Lett. B 594, 171 (2004).

[85] E. Cattaruzza, A. Del Fabbro, and D. Treleani, Phys. Rev. D 72, 034022 (2005).

[86] J. R. Gaunt and W. J. Stirling, J. High Energy Phys. 03 (2010) 005.

[87] PYTHIA8 allows to generate always two hard interactions in a given event by setting SecondHard: generate=on. However, usage of this flag together with Angantyr is not supported and will lead to wrong results.

[88] Namely, it accounts only for a gluon splitting of a type $g \rightarrow q \bar{q}$; for details, see [74].

[89] M. Diehl, J. R. Gaunt, and K. Schönwald, J. High Energy Phys. 06 (2017) 083.

[90] M. Diehl and J. R. Gaunt, Adv. Ser. Dir. High Energy Phys. 29, 7 (2018).

[91] W. Busza, J. E. Elias, D. F. Jacobs, P. A. Swartz, C. C. Young, and M. R. Sogard, Phys. Rev. Lett. 34, 836 (1975).

[92] A. Bialas, M. Bleszynski, and W. Czyz, Nucl. Phys. B111, 461 (1976).

[93] N. N. Nikolaev and S. Pokorski, Phys. Lett. 80B, 290 (1979).

[94] N. N. Nikolaev, Usp. Fiz. Nauk 134, 369 (1981) [Sov. Phys. Usp. 24, 531 (1981)].

[95] In our simulations, we choose a pseudorapidity axis to run in direction from a nucleus to a proton.

[96] B. Blok, Yu. Dokshitser, L. Frankfurt, and M. Strikman, Eur. Phys. J. C 72, 1963 (2012).

[97] M. G. Ryskin and A. M. Snigirev, Phys. Rev. D 83, 114047 (2011).

[98] M. G. Ryskin and A. M. Snigirev, Phys. Rev. D 86, 014018 (2012).

[99] J. R. Gaunt, J. High Energy Phys. 01 (2013) 042.

[100] A. V. Manohar and W. J. Waalewijn, Phys. Lett. B 713, 196 (2012).

[101] B. Cabouat, J. R. Gaunt, and K. Ostrolenk, J. High Energy Phys. 11 (2019) 061.

[102] D. Binosi and L. Theussl, Comput. Phys. Commun. 161, 76 (2004).

[103] B. Gough, GNU Scientific Library Reference Manual, 3rd ed. (Network Theory Ltd., Bristol, UK, 2009).

[104] G. P. Lepage, J. Comput. Phys. 27, 192 (1978).

[105] J. D. Hunter, Comput. Sci. Eng. 9, 90 (2007).

[106] P. Virtanen et al., Nat. Methods 17, 261 (2020).

[107] T. Oliphant, Guide to NumPy (Trelgol Publishing, Spanish Fork, UT, USA, 2006).

[108] A. Buckley, J. Ferrando, S. Lloyd, K. Nordström, B. Page, M. Rüfenacht, M. Schönherr, and G. Watt, Eur. Phys. J. C 75, 132 (2015).

[109] A. D. Martin, W. J. Stirling, R. S. Thorne, and G. Watt, Eur. Phys. J. C 63, 189 (2009). 